\title{
Square Van Atta reflector with conducting mounting flame
}

\author{
Nielsen, Erik Dragø
}

Published in:

I E E E Transactions on Antennas and Propagation

Publication date:

1970

Document Version

Publisher's PDF, also known as Version of record

Link back to DTU Orbit

Citation (APA):

Nielsen, E. D. (1970). Square Van Atta reflector with conducting mounting flame. I E E E Transactions on Antennas and Propagation, 18(1), 48-54.

\section{General rights}

Copyright and moral rights for the publications made accessible in the public portal are retained by the authors and/or other copyright owners and it is a condition of accessing publications that users recognise and abide by the legal requirements associated with these rights.

- Users may download and print one copy of any publication from the public portal for the purpose of private study or research.

- You may not further distribute the material or use it for any profit-making activity or commercial gain

- You may freely distribute the URL identifying the publication in the public portal

If you believe that this document breaches copyright please contact us providing details, and we will remove access to the work immediately and investigate your claim 
Research Projects Agency and the National Science Foundation under contract with the Air Force Office of Scientific Research.

\section{REFERENCES}

[1] R. C. Spencer, C. J. Sletten, and J. E. Walsh, "Correction of spherical aberration by a phased line source," Proc. 1949 $N E C$ (Chicago, Ill.), vol. 5 , p. 320 .

[2] A. F. Kay, "A line source feed for a spherical reflector," Contract AF 19(604)-5532, AFCRL 529, May 1961.

[3] A. W. Love, "Spherical reflecting antennas with correcting line sources," IEEE Trans. Antennas and Propagation, vol. AP-10, pp. 529-537, September 1962.

[4] A. C. Schell, "Diffraction theory of large-aperture spherical reflector antennas," IEEE Trans. Antennas and Propagation, vol. AP-11, pp. 428-432, July 1963.

[5] F. S. Holt and E. L. Bouche, "A Gregorian corrector for spherical reflectors," IEEE Trans. Antennas and Propagation, vol. AP-12, pp. 44-47, January 1964.

[6] G. Hyde, "Focal region fields of a spherical reflector RCA," AFCRL-66-48, Contract AF 19(628)-2758, January 1966.

[7] G. C. MeCormick, "A line feed for a spherical reflector," IEEE Trans. Antennas and Propagation, vol. AP-15, pp. 639 645, September 1967.

[8] A. W. Love and J. J. Gustincic, "Line source feed for a spherical reflector," IEEE Trans. Antemnas and Propagation (Communications), vol. AP-16, pp. 132-134, Januar. 1968.

[9] M. H. Cohen and G. E. Perona, "A correcting feed at $611 \mathrm{MHz}$ for the AIO reflector," IEEE Trans. Antennas and Propagation (Communications), vol. AP-15, pp. 482-4\&3, May 1967.

[10] "Report of the ad hoc advisory panel for large radio astronomy facilities," NSF, Washington, D. C. . August 14, 1967.

[11] J. Ruze, in Antenna Engineering Handbook, H. Jasik, Ed. New York: McGraw-Hill, 1961, p. 2-39.

[12] K. I. Kellermann, I. I. K. Pauliny-Toth, and P. J. S. Williams, "The spectra of radio sources in the revised 3C catalog," $A P J$, vol. $15 \bar{i}$, pp. 1-34, July 1969.

\title{
Square Van Atta Reflector with Conducting Mounting Plane
}

\author{
ERIK DRAGØ NIELSEN
}

\begin{abstract}
A}$ theoretical and numerical analysis of square Van Atta reflectors has been carried out with or without a conducting plate, used for mounting of the antenna elements. The Van Atta reflector investigated has antenna elements which are parallel half-wave dipoles interconnected in pairs by transmission lines of equal electrical length. The dipoles are placed in a plane which is parallel to the conducting plate when this is present. In the theory, each pair of antenna elements with the interconnecting transmission line is represented by an equivalent circuit. The mutual impedance between the antenna elements and the reradiation from the elements as well as from the conducting plate have been taken into account. The influence of the conducting plate on the induced dipole currents has been treated using the theory of images. The scattering cross section of Van Atta reflectors with or without a conducting plate has been examined numerically using an electronic computer, and some of the results have been compared with experimental results obtained by others.
\end{abstract}

\section{Introduction}

$\mathbf{I}$ $T$ PREVIOUS papers on the theoretical analysis of Van Atta reflectors, Appel-Hansen [1] has considered linear Van Atta reflectors consisting of four parallel halfwave dipoles and Larsen [2] has given an analysis of arbitrary Van Atta reflectors consisting of dipoles. Except for these two papers, no direct and detailed theoretical

Manuscript received April 17, 1969; revised August 18, 1969. This research was supported in part by the Air Force Cambridge Research Laboratories (CRDG), L. G. Hanscom Field, Bedford, Mass., through the European Office of Aerospace Research (OAR), U. S. Air Force, under Contract AF 61(052)-794.

The author is with the Laboratory of Electromagnetic Theory, Technical University of Denmark, Lyngby, Denmark. investigation of this particular type of reflector has yet been published. However, a number of papers have appeared describing experimental investigations and limited analytical treatments of the Van Atta reflector.

In addition to the papers referenced in [1] and [2], two papers by Appel-Hansen [3] and Larsen [4] describe further details of the theoretical approach and experimental results. A communication between Kurss, Kahn, Appel-Hansen, and Larsen [5] discusses the results of [1] and [2] in relation to an earlier communication by Furss and Kahn [6]. Furthermore, Appel-Hansen has carried out an experimental investigation of linear and square Van Atta reflectors backed by conducting plates [7]. However, the size of the square Van Atta reflector was so small, in wavelengths, that a comparison between those measured results and the numerical results of the present paper would not be reasonable because of the assumptions made in the theory.

Mounting of the dipole elements of a Van Atta reflector above a perfectly conducting ground plane parallel to the plane of dipoles is the most effective method used in experiments for reduction of the field scattered from the transmission lines or reflected from the background, but it has not been considered theoretically until the work described in this paper. The theory applied in the present investigation was first suggested by Larsen [4]. It is based on and very close to the theory previously discussed in [2], and where possible and convenient, a reference will be given rather than repetition of equations of minor impor- 
tance. Thus, the purpose of the present paper is to describe, by modifications to the theory previously developed by Larsen [2], an analytical and numerical treatment of square Van Atta reflectors with a conducting plate. The results of such an investigation are most useful in connection with the design of Van Atta reflectors for practical use, where a conducting plate will usually be necessary for obtaining a good performance of the reflector.

A comparison is made between the numerical results of this investigation and the experimental results obtained by Sharp [8] for a square Van Atta reflector consisting of 16 dipole elements mounted parallel to and a quarter of a wavelength above a conducting plate.

Further, the scattering cross section of square Van Atta reflectors has been calculated, and the influence of the distance between adjacent elements, the distance between the elements and the plate, and the length and the characteristic impedance of the transmission lines is discussed.

\section{THEORY}

The system investigated is shown in Fig. 1. The antenna elements are half-wave dipoles placed in the $x y$ plane and parallel to the $y$ axis in a square configuration. A square perfectly conducting plate is placed parallel to and at a distance $h$ below the $x y$ plane.

A problem involving a conducting plate lends itself to treatment by the theory of images. However, this theory requires a conducting plate of infinite extent, which was not present in the actual problem. Furthermore, it was desirable to use the same procedure and the same computer program for reflectors with a conducting plate as well as for reflectors without a plate. This led to an approach similar to the theory of images. The course of the computations has been chosen such that it follows the method of investigation described in [2].

The total voltages induced in the image dipole elements of the reflector are equal to the voltages in the real dipoles but of opposite sign. This means that the currents in corresponding elements will also be equal but of opposite sign.

The equations given by Larsen [2] connecting the unknown antenna currents and induced voltages through the mutual impedance matrix will thus be changed to the following, respectively.
The notation $z_{r s}{ }^{\prime}$ means the mutual impedance between element number $r$ of the dipole array and element number $s$ of the image dipole array. All quantitiesin these equations are normalized as in [2].

\section{A. Induced Voltages in Dipoles}

The open circuit voltage induced in the dipoles will be given by

$$
V=\bar{E} \cdot \bar{L}_{\mathrm{eff}}
$$

where $\bar{E}$ is the electric field strength at the position of the element and $\vec{L}_{\text {eff }}$ is the effective length of a half-wave dipole element.

The electric field strength is found from ordinary reflection theory. The primary plane wave is decomposed into two plane waves, one polarized in the plane of incidence, and the other perpendicular to the plane of incidence. Each of these give rise to two components of field strength in the plane of dipoles, one from the primary wave, and one from the reflected wave.

Thus the following expression is obtained for the open circuit voltage in the half-wave dipoles:

$$
\begin{aligned}
V= & E_{0} \frac{2 \lambda}{\pi} \frac{\cos [(\pi / 2) \cos u]}{\sin u}\left(\cos v \cos \theta_{i} \sin \phi_{i}-\sin v \cos \phi_{i}\right) \\
& \cdot \sin \left(k h \cos \theta_{i}\right) \exp \left[i\left(\pi / 2+k h \cos \theta_{i}\right)\right] \\
& \cdot \exp \left[-i k \sin \theta_{i}\left(x \cos \phi_{i}+y \sin \phi_{i}\right)\right]
\end{aligned}
$$

where $h$ is the distance from the plane of dipoles to the conducting plate, and $\left(\theta_{i}, \phi_{i}\right)$ determines the direction of incidence of the primary plane wave, as shown in Fig. 1. The parameter $u$ is the angle between the direction of the dipole and the direction of propagation of the primary plane wave. From the notation, it is found that $\cos u=$ $\sin \theta_{i} \sin \phi_{i}$.

\section{B. Mutual Impedances Between Dipoles}

The self-impedance of the dipoles and the mutual impedance between them is found by using the induced EMF method under the assumption of sinusoidal current distribution on the dipoles.

The self-impedance of element $i$ is called $z_{i i}$ and $z_{i j}$ is the mutual impedance between the $i$ th and the $j$ th element.

$$
\begin{aligned}
& \left(v_{m}-v_{k}\right) \cos (k a / 2)=\left\{-i \sin (k a / 2)+\left(z_{m m}-z_{m k}\right) \cos (k a / 2)\right\}\left(i_{m}-i_{k}\right) \\
& +\sum_{n=1, n \neq m, k}^{N} i_{n}\left(z_{n m}-z_{n k}\right) \cos (k a / 2)-\sum_{n=1, n \neq m m, k}^{N} i_{n}\left(z_{n m^{\prime}}-z_{n k^{\prime}}\right) \cos (k a / 2) \\
& \left(v_{m}+v_{k}\right) \sin (k a / 2)=\left\{i \cos (k a / 2)+\left(z_{m m}+z_{m k}\right) \sin (k a / 2)\right\}\left(i_{m}+i_{k}\right) \\
& +\sum_{n=1, n \neq m, k}^{N} i_{n}\left(z_{n m}+z_{n k}\right) \sin (k a / 2)-\sum_{n=1, n \neq m, k}^{N} i_{n}\left(z_{n m}{ }^{\prime}+z_{n k}{ }^{\prime}\right) \sin (k a / 2) \\
& v_{p} \cos k a=i_{p}\left(z_{p p} \cos k a-i \sin k a\right)+\sum_{n=1, n \not \neq p}^{N} i_{n} z_{n p} \cos k a-\sum_{n=1, n \neq p}^{N} i_{n} z_{n p}{ }^{\prime} \cos k a .
\end{aligned}
$$




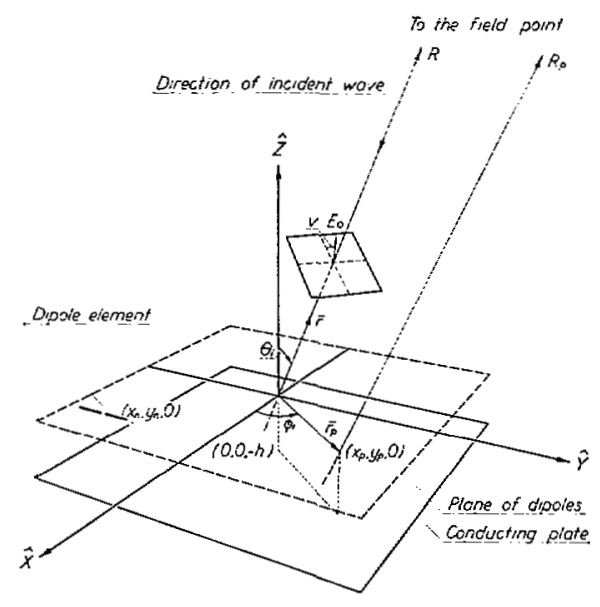

Fig. 1. Geometry of reflector system.

Using the induced EMF method, the following expression is obtained for the mutual impedance $z_{i j}$ between two dipoles of length $l_{i}$ and $l_{j}$ :

$$
z_{i j}=-\frac{1}{Z_{0}} \int_{0}^{l_{i}} \frac{I_{i}(z) E_{i j}(z)}{I_{i} I_{j}} d z
$$

and, for the self-impedance of a dipole of length $l_{i}$,

$$
z_{i i}=-\frac{1}{Z_{0}} \int_{0}^{l_{i}} \frac{I_{i}(z) E_{i}(z)}{I_{i}^{2}} d z
$$

where the mutual and self-impedances are normalized with respect to the characteristic impedance $Z_{0}$ of the transmission lines. $I_{i}$ is the terminal current of antenna $i, I_{i}(z)$ is the current distributed along this antenna, $E_{i j}(z)$ is the electrical field strength at and parallel to antenna $i$ due to the current in antenna $j$, and $E_{i}(z)$ is the electrical field strength at and parallel to the $i$ th antenna due to its own current.

Using these formulas, the mutual impedances between all antenna elements, real dipoles as well as their images, have been calculated, assuming that all elements not under consideration have been removed. This approximation has been tested by Davies [9] and may be expected to give good results when the spacing between the dipoles is as great as is the case for Van Atta reflectors, and the largest dimension of the system of dipoles is not much larger than approximately three wavelengths.

When the self and mutual impedances are known, a linear system of complex equations based on (1)-(3) may be set up for determination of the complex currents in the dipole elements, real dipoles as well as image dipoles, similar to the system of equations found in [2] for the real dipoles only.

\section{Field Reradiated from System of Dipoles}

When the currents are found, the electric field reradiated from the dipoles and their images is given by

$$
\bar{E}_{\text {reilector }}=\bar{E}_{\text {ref }}(R, \theta, \phi)\left[G_{\text {dipole }}(\theta, \phi)+G_{\text {image }}(\theta, \phi)\right]
$$

where the reflector is considered as an antenna composed of two identical groups of dipoles: the group of real dipoles and the group of image dipoles. Here

$$
\begin{array}{r}
\bar{E}_{\mathrm{ref}}(R, \theta, \phi)=\frac{i \zeta_{0} E_{0}}{\pi \cdot Z_{0}} \frac{\exp (i k R)}{k R} \frac{\cos [(\pi / 2) \sin \theta \sin \phi]}{\left(1-\sin ^{2} \theta \sin ^{2} \phi\right)^{1 / 2}} \\
\cdot[\sin \phi \cos \theta \hat{\theta}+\cos \phi \hat{\phi}]
\end{array}
$$

is the reradiated electric field at a point in the distance $R$ from a reference antenna in $(0,0,0)$.

The array factor for each group of dipoles considered, may be cxpressed as

$$
G(\theta, \phi)=\sum_{n=1}^{N} i_{n} \exp \left(-i k \bar{r}_{n} \cdot \hat{r}\right)
$$

where $N$ is the number of antenna elements in the array, $i_{n}$ is the total complex current in the $n$th dipole, $\bar{r}_{n}$ is the radius vector from the point of reference $(0,0,0)$ to the $n$th antenna, and $\hat{r}$ is a unit vector in the direction from $(0,0,0)$ to the field point.

Introducing the unit vector

$$
\hat{r}=\hat{x} \sin \theta \cos \phi+\hat{y} \sin \theta \sin \phi+\hat{z} \cos \theta
$$

and the radius vectors of the $n$th dipole and the $n$th image dipole

$$
\begin{aligned}
& \bar{r}_{n, \mathrm{~d} \text { jole }}=\left(x_{n} \hat{x}, y_{n} \hat{y}, 0\right) \\
& \bar{r}_{n, \mathrm{image}}=\left(x_{n} \hat{x}, y_{n} \hat{y},-2 h \hat{z}\right)
\end{aligned}
$$

and, remembering that the currents of the image dipoles are equal to the currents of the real dipoles, but of opposite sign, (8) yields

$$
\begin{aligned}
\bar{E}_{\text {rếlector }}= & \bar{E}_{\text {ref }}(R, \theta, \phi) \sum_{n=1}^{N} i_{n}\left\{\operatorname { e x p } \left[-i k\left(x_{n} \sin \theta \cos \phi\right.\right.\right. \\
& \left.\left.+y_{n} \sin \theta \sin \phi\right)\right]-\exp \left[-i k\left(x_{n} \sin \theta \cos \phi\right.\right. \\
& \left.\left.\left.+y_{n} \sin \theta \sin \phi-2 h \cos \theta\right)\right]\right\}
\end{aligned}
$$

\section{Field Reradiated from Square Conducting Ground Plate}

In order to find the field reflected from the plate itself, use is made of the method of physical optics given, e.g., by Kerr $[10]$, where the backscattering cross section of a rectangular plate is given for a plate, the dimensions of which are not small compared to the wavelength. The reflecting properties of the plate are assumed not to be influenced by the presence of the dipoles.

The reradiated field is found from the surface current distribution $\bar{K}$ on the plate. This current is assumed to be the same as if the plate were infinite in extent.

Under this assumption, the total tangential magnetic field at the plate is

$$
\begin{aligned}
\bar{H}_{\mathrm{tot}}{ }^{t}= & \left(2 E_{0} / \zeta_{0}\right) \exp \left[i k h \cos \theta_{i}\right] \\
& \cdot \exp \left[-i k \sin \theta_{i}\left(x_{p} \cos \phi_{i}+y_{p} \sin \phi_{i}\right)\right] \\
& \cdot\left[\hat{x}\left(-\cos v \sin \phi_{i}+\sin v \cos \theta_{i} \cos \phi_{i}\right)\right. \\
& \left.+\hat{y}\left(\cos v \cos \phi_{i}+\sin v \cos \theta_{i} \sin \phi_{i}\right)\right]
\end{aligned}
$$


and the surface current is

$$
\bar{K}=\hat{z} \times \bar{H}_{\text {tot }}{ }^{t} .
$$

(An explanation of the notation may be found in Fig. 1.)

The vector potential of the surface current is

$$
\bar{A}=\frac{\mu}{4 \pi} \int_{\text {plate }} \bar{K} \frac{\exp \left(i k R_{p}\right)}{R_{p}} d \alpha
$$

where $R_{p}$ is the distance from the plate to the field point as shown in Fig. 1.

The electric field reflected from the plate may be expressed as

$$
\bar{E}_{\text {plate }}=i \omega \bar{A} .
$$

By using the far field approximations for $R_{p}$ in (15), which, according to Fig. 1 , means $R_{p} \cong R-\bar{r}_{p} \cdot \hat{r}+h \cos \theta$ in the numerator and $R_{p} \cong R$ in the denominator, the following expression is obtained for the field radiated from the plate:

$$
\bar{F}_{\text {plate }}(R, \theta, \phi)=\frac{i \omega \mu E_{0}}{2 \pi \zeta_{0}} \frac{\exp (i k R)}{R} \exp \left[i k h\left(\cos \theta_{i}+\cos \theta\right)\right]
$$$$
\cdot b^{2} \frac{\sin \alpha}{\alpha} \frac{\sin \beta}{\beta}\left\{\hat { \theta } \left[-\left(\cos v \cos \phi_{i}+\sin v\right.\right.\right.
$$

$\left.\cdot \cos \theta_{i} \sin \phi_{i}\right) \cos \phi \cos \theta+(-\cos v$

$\left.\cdot \sin \phi_{i}+\sin v \cos \theta_{i} \sin \phi_{i}\right) \sin \phi$

$\cdot \sin \theta]+\hat{\phi}\left[\left(\cos v \cos \phi_{i}+\sin v\right.\right.$

$\left.\cdot \cos \theta_{i} \sin \phi_{i}\right) \sin \phi+\left(-\cos v \sin \phi_{i}\right.$

$\left.\left.\left.+\sin v \cos \theta_{i} \cos \phi_{i}\right) \cos \phi\right]\right\}$

where

$$
\begin{aligned}
& \alpha=\frac{1}{2} k b\left(\sin \theta_{i} \cos \phi_{i}+\sin \theta \cos \phi\right) \\
& \beta=\frac{1}{2} k b\left(\sin \theta_{i} \sin \phi_{i}+\sin \theta \sin \phi\right)
\end{aligned}
$$

and $b$ is the side length of the square conducting plate.

\section{E. Total Reradiated Field}

The total electric field $\bar{E}_{\text {total }}$ reradiated from the reflector system consisting of the simple Van Atta reflector and the conducting plate may be found by superposition of the electric field reflected from the plate (17) and the electric field reradiated from the Van Atta reflector and its image (12) :

$$
\bar{E}_{\text {total }}(R, \theta, \phi)=\bar{E}_{\text {plate }}(R, \theta, \phi)+\bar{E}_{\text {reflector }}(R, \theta, \phi) .
$$

When describing reradiation or scattering properties, the most commonly used quantity is the differential scattering cross section, which, in an arbitrary direction and independent of the distance, gives the far field scattered from a target for an incident plane wave.

The differential scattering cross section for a Van Atta reflector with conducting plate is given by

$$
\sigma(\theta, \phi)=4 \pi R^{2} \frac{\bar{S}_{r}(\theta, \phi) \cdot \hat{n}_{r} \mid}{\left|\bar{S}_{i}(\theta, \phi) \cdot \hat{n}_{i}\right|}=4 \pi R^{2} \frac{E_{0}{ }^{2}}{E_{\text {total }} !^{2}}
$$

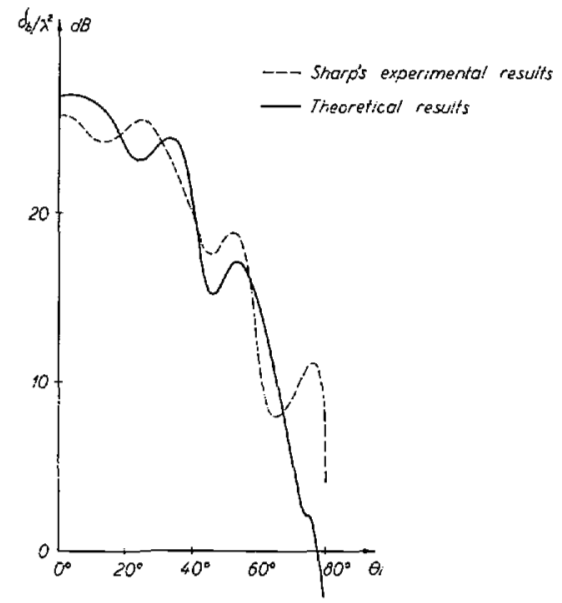

Fig. 2. Normalized backscattering cross section of 16-element square Van Atta reflector with conducting plate. $a=0.41 \lambda, d=$ $0.6 \lambda, h=0.25 \lambda, Z_{0}=73 \mathrm{ohms}, \phi_{i}=0^{\circ}, v=90^{\circ}$.

where $\bar{S}_{r}$ and $\bar{S}_{i}$ are the Poynting vectors of the reflected and incident fields, and $\hat{n}_{r}$ and $\hat{n}_{i}$ are unit vectors in the direction of propagation of the reflected and incident fields, respectively. $E_{0}$ is the amplitude of the incident wave and $\bar{E}_{\text {total }}$ is the reradiated electric field found from (20).

In the numerical investigations discussed in the next section, the electric field reradiated from the Van Atta reflector with or without the conducting plate will be described by the normalized scattering cross section $\sigma(\theta, \phi) / \lambda^{2}$. In particular, the backscattering cross section $\sigma_{b} / \lambda^{2}=\sigma\left(\theta_{i}, \phi_{i}\right) / \lambda^{2}$ of the reflector will be discussed.

\section{Numerical Investigation}

\section{A. Comparison with Results Obtained by Sharp}

As mentioned previously, Sharp [8] has made an experimental investigation of a square Van Atta reflector consisting of 16 half-wave dipoles mounted parallel to and a quarter-wavelength above a conducting plate. The dipoles were set up in a four-by-four array with a spacing of 0.6 wavelength. A number of measurements were performed for various polarizations of the incident plane wave and at various frequencies.

The comparison between this experimental reflector and the Van Atta reflector investigated here has been carried out by considering the backscattering cross section $\sigma_{b} / \lambda^{2}$ of the reflector in the $x z$ plane $\left(\phi_{i}=0^{\circ}\right)$, when the incident plane wave is polarized parallel to the dipoles. From the report made by Sharp, the curve denoted "array" in pattern 1 has been redrawn in Fig. 2 of this paper. In the same figure, the computed values of $\sigma_{b} / \lambda^{2}$ are shown for a reflector with the same parameters as Sharp's model. The frequency used by Sharp was $2850 \mathrm{MHz}$ corresponding to a wavelength $\lambda=10.519 \mathrm{~cm}$. The interconnecting transmission lines were of length $22.5 \pm 0.1$ inches, which corresponds to the electrical length $5.43 \lambda \pm 0.02 \lambda$. In order to obtain matching conditions between the dipoles and the transmission lines, the characteristic impedance of the lines is $Z_{0}=73$ ohms. 

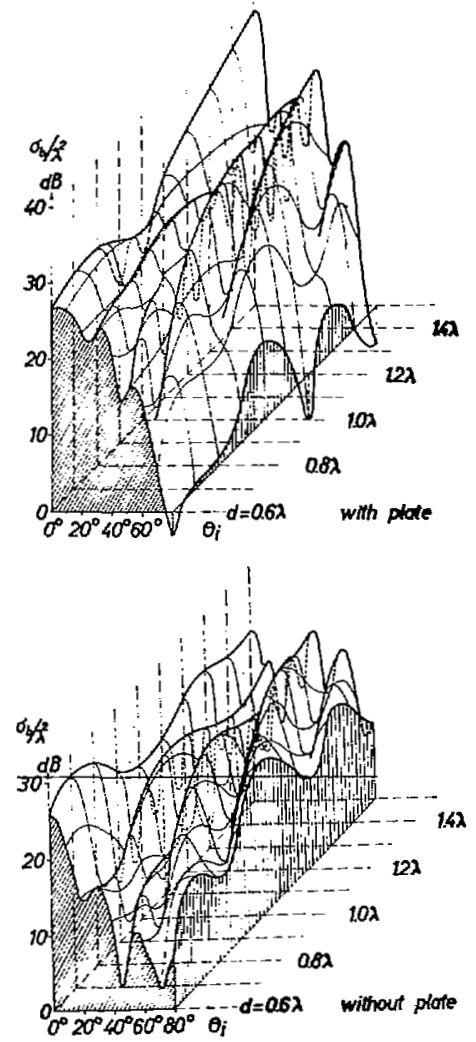

Fig. 3. Normalized backscattering cross section for the reflector of Fig. 2 as a function of the distance $d$ between the dipoles.

Because of the uncertainty in the actual electrical length of the transmission lines of the experimental reflector, a number of reflectors with various lengths of the lines have been examined theoretically. It turned out that transmission lines of length $a=0.41 \lambda$ gave the reradiation pattern which was closest to the one measured by Sharp. This value is in accordance with the value given for the experimental reflector $(0.43 \lambda \pm 0.02 \lambda)$.

From the two curves in Fig. 2, it is seen that, for angles of incidence $\theta_{i}$ less than $60^{\circ}$, the backscattering cross section of the experimental and theoretical reflector differ with an amount of at most $2.6 \mathrm{~dB}$. When $\theta_{i}$ is larger than $60^{\circ}$, the edge of the conducting plate will influence the reradiated field to such a degree that a comparison between the measured and computed results would not be reasonable.

To some extent, the discrepancies between the two curves of Fig. 2 are probably due to inaccuracies in the experiments. The curves denoted "flat plate" in patterns 1 and 2 of Sharp's report show the measured values of $\sigma_{b} / \lambda^{2}$ for the same square conducting plate obtained during two different measurements. It is seen that the two curves for $\theta_{i}$ less than $60^{\circ}$ deviate with about $2.0-2.5 \mathrm{~dB}$, and that there is an angular displacement of the curves of about $3^{\circ}-9^{\circ}$. This must be due to uncertainty of the measurements, and it is seen that the theoretical and experimental curves of Fig. 2 agree within the limits of this uncertainty for angles of incidence less than $60^{\circ}$.

A comparison between Sharp's measured backscattering cross section for the plate alone and our computed results

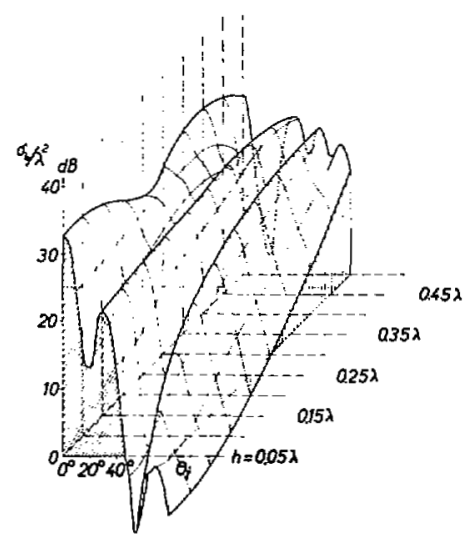

Fig. 4. Nurmalized backscattering cross section for the reflector of Fig. 2 as a function of the distance $h$ between the dipoles and the plate.

shows a good agreement when the angle of incidence $\theta_{i}$ is less than $60^{\circ}$, so that the edges of the plate do not. affect the reradiated field to a noticeable extent.

\section{B. Effect of Changing the Parameter Values}

The parameters of the Van Atta reflector are

$N$ number of elements

$d$ distance between adjacent elements

$h$ distance between the elements and the plate

$Z_{u}$ characteristic impedance of the transmission lines

a length of the transmission lines.

In this section, we will investigate what happens to the backscattering cross section $\sigma_{b} / \lambda^{2}$ of the reflector, when some of these parameters are varied in turn. The incident field is polarized parallel to the dipoles and $\phi_{i}=0$. As a starting point for the variations, the reflector which was compared with Sharp's experimental model in Section III-A will be considered with and without a conducting plate. The initial values of the parameters will thus be $N=16$ elements, $d=0.6 \lambda, h=0.25 \lambda, Z_{0}=73 \mathrm{ohms}$ and $a=0.41 \lambda$. The size of the square conducting plate is $N \cdot d^{2}$.

In Fig. 3, the distance between adjacent dipoles is varied from $d=0.6 \lambda$ to $d=1.5 \lambda$. It will be noticed that $\sigma_{b} / \lambda^{2}$, in the direction normal to the reflector $\left(\theta_{i}=0^{\circ}\right)$, has a minimum when $d=1.0 \lambda$. This minimum is most pronounced when the conducting plate is taken into account, but occurs in the case of the simple reflector, too. When $d$ increases, it appears that the irregularities of the curve of $\sigma_{b} / \lambda^{2}$ inerease but it also turns out that the level of backscattering for $\theta_{i}$ larger than $40^{\circ}$ is increased essentially. Since the coupling between the dipoles decreases for increasing $d$, the latter is in agreement with the fact that coupling usually makes the minimum value of $\sigma_{b} / \lambda^{2}$ decrease. This is in accordance with what has been found for a linear four-element Van Atta reflector [11].

Changing the distance $h$ from the dipoles to the conducting plate, from $h=0.05 \lambda$ to $h=0.5 \lambda$ results in the curve of $\sigma_{b} / \lambda^{2}$ shown in Fig. 4. It is seen that the backscattering in the direction $\theta_{i}=0^{\circ}$ obtains its smallest value when $h=0.25 \lambda$, which is the distance Sharp used 

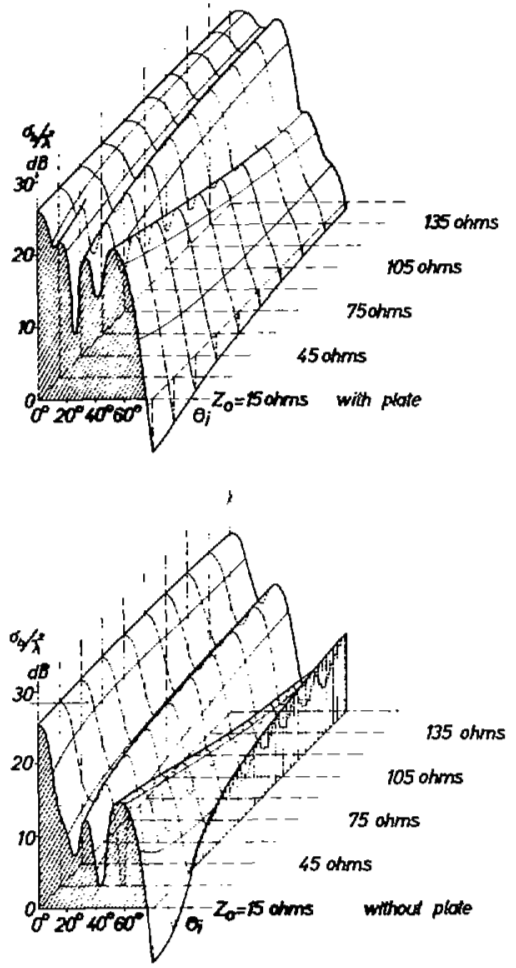

Fig. 5. Normalized backscattering cross section for the reflector of Fig. 2. as a function of the characteristic impedance $Z_{0}$ of the transmission line.

in his experimental model. From Fig. 4, it appears that a higher level of backseattering for all angles of incidence might have been obtained if $h=0.35 \lambda$ had been chosen in the experimental model instead of $h=0.25 \lambda$.

The characteristic impedance of the transmission lines is varied from $Z_{0}=15 \mathrm{ohms}$ to $Z_{0}=150 \mathrm{ohms}$ and the results are shown in Fig. 5 . When the plate is not taken into account, it turns out that $\sigma_{b} / \lambda^{2}$ increases for $0^{\circ}<\theta_{i}<$ $45^{\circ}$, decreases for $40^{\circ}<\theta_{i}<70^{\circ}$, and again increases for $\theta_{i}>70^{\circ}$, when the characteristic impedance $Z_{0}$ is increased up to $150 \mathrm{ohms}$. For $\theta_{i}$ equal to $0^{\circ}, 45^{\circ}$, and $70^{\circ}$, the value of $\sigma_{b} / \lambda^{2}$ is almost unchanged. When the conducting plate is taken into account, the same occurs except for the range $70^{\circ}<\theta_{i}<80^{\circ}$, where $\sigma_{b} / \lambda^{2}$ now is practically unchanged. It is seen that in this way the curve of $\sigma_{b} / \lambda^{2}$ obtains a smoother shape for larger values of $Z_{0}$, especially when the plate is taken into account. At the same time, unfortunately, $\sigma_{b} / \lambda^{2}$ decreases much faster for increasing $\theta_{i}$.

In Fig. 6, the length of the transmission lines is varied from $a=0.0 \lambda+p \lambda$ to $a=1.0 \lambda+p \lambda$, where $p$ is an integer. When the plate is not taken into account, it turns out that $\sigma_{b} / \lambda^{2}$, in the direction normal to the reflector, tends to zero when $a=p \lambda$, whereas it has a maximum for $a=0.5 \lambda+p \lambda$. This is in accordance with the results obtained by Appel-Hansen [1] for a linear Van Atta reflector. Furthermore, the curve has a deep minimum in the direction $\theta_{i}=57^{\circ}$ when $a=0.5 \lambda+p \lambda$. When the conducting plate is taken into account, the minimum of $\sigma_{b} / \lambda^{2}$ for $a=p \lambda$ in the normal direction is changed to a maximum, whereas the maximum for $a=0.5 \lambda+p \lambda$ is
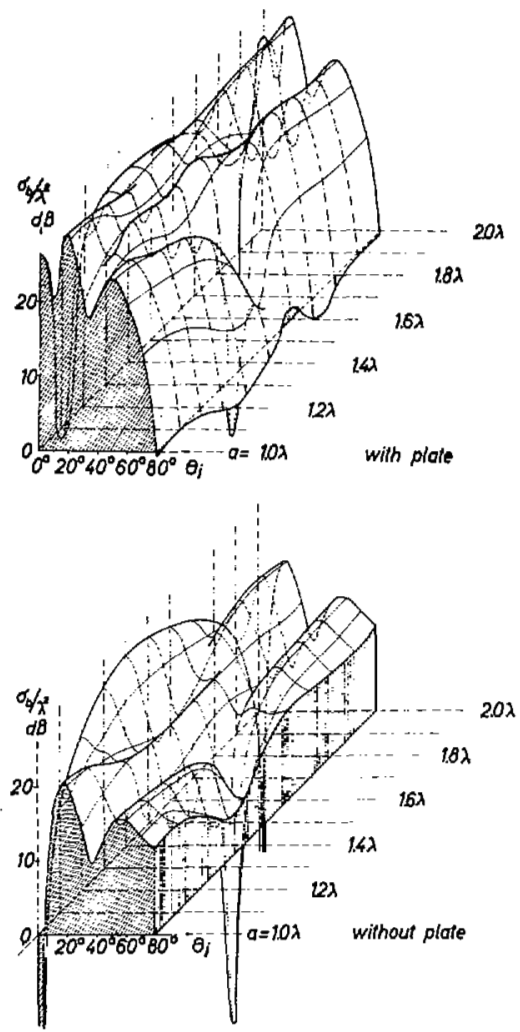

Fig. 6. Normalized backscattering cross section for the reflector of Fig. 2 as a function of the length $a$ of the transmission lines.

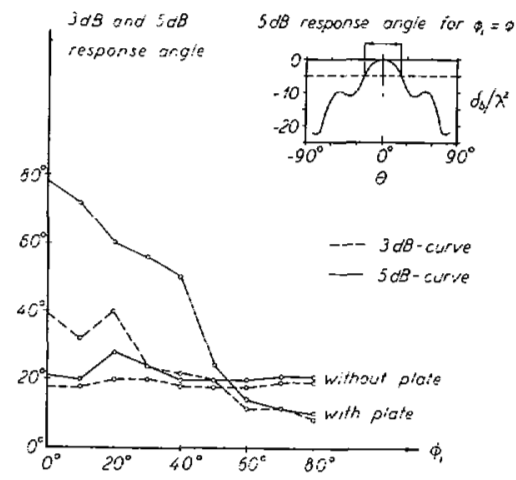

Fig. 7. Response angle coverage for 16-element Van Atta reflector with or without a plate.

unchanged. Further, two new minima occur for $a=0.1 \lambda+$ $p \lambda$ and $a=0.9 \lambda+p \lambda$, respectively, and the deep minimum in the direction $\theta_{i}=5 \bar{\sigma}^{\circ}$ when $a=0.5 \lambda+p \lambda$ is essentially reduced. It is seen that the length of the lines chosen by Sharp in his experimental investigation, i.e., $a=0.43 \lambda$

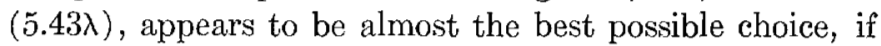
maximum of $\sigma_{b} / \lambda^{2}$ in the normal direction and a smoothly shaped curve is wanted.

\section{Effect of Turning the Plane of Incidence}

The plane of incidence is turned by gradually changing $\phi_{i}$ from 0 to $90^{\circ}$. The incident plane wave is polarized in a direction parallel to the plane of the dipoles and perpendicular to the plane of incidence.

The curves in Fig. 7 have been drawn as a measure of the angular coverage of the reflector. They show the angular 
range in $\theta$ over which the backscattering cross section decreases 3 and $5 \mathrm{~dB}$, respectively, below the maximum value. These angles are called the $3-\mathrm{dB}$ response angle and the $\bar{J}-\mathrm{dB}$ response angle, respectively, and its definition is shown in the small figure at the top of Fig. 7 . From the curves, it appears that the Van Atta reflector with a conducting plate shows a larger response angle than the reflector without plate, over an angular range from $\phi_{i}=0^{\circ}$ to $\phi_{i}=50^{\circ}$. Although the calculated response angles show fluctuations as a function of $\phi_{i}$, it can be stated that the average $\tilde{5}-\mathrm{dB}$ response angle of the reflector with a conducting plate is almost twice as large as that of the dipole reflector alone in the angular range between $\phi_{i}=0^{\circ}$ and $\phi_{i}=45^{\circ}$. For the $3-\mathrm{dB}$ response angle, the corresponding angular range is between $\phi_{i}=0^{\circ}$ and $\phi_{i}=30^{\circ}$.

\section{Concltision}

Arbitrary Van Atta reflectors with or without a conducting plate have been investigated analytically and numerically.

If the plate is present, the reflecting system consists of two devices, the dipoles and the plate. The system of dipoles is then treated along the same lines as in [2] , but the presence of the plate will influence the dipole system in such a way that the induced voltage, the mutual impedance matrix, and the determination of the reradiated field have to be changed. The reflecting properties of the plate are supposed not to be affected by the shadowing effect of the dipoles.

The numerical investigation shows a good agreement with an experimental reflector investigated by Sharp [8]. The effect of varying the parameters of the reflector has been investigated by considering the backscattering cross section of several different reflectors, with or without a conducting plate. From an examination of the results obtained, it turns out that the presence of a conducting plate at a distance $h$ below the dipole system will cause both advantages and disadvantages. Among the former, the most pronounced is a rise of the level of backscattering in the direction normal to the plane of the reflector, and a highly increased 5- $\mathrm{dB}$ response angle, while the largest disadvantage is that the backscattering cross section decreases very fast when the direction of incidence makes an angle $\theta_{i}$ larger than $60^{\circ}$ with the normal to the reflector. Further, it turns out that, by choosing proper values of the parameters, the reradiation pattern may be changed to compare better with a prescribed form, and that the values of the parameters used in Sharp's experimental model are not the best possible in order to obtain a Van Atta reflector that behaves as stated in the patent description.

Probably, results which compare better with the experimental results measured by Sharp may be obtained using a theory for the field reflected from the conducting plate other than the physical optics theory used here. This theory may be Keller's geometrical theory of diffraction which, in contrast to the theory used, will take into account the scattering of the incident field about the edges of the conducting plate.

\section{ACKNOWLEDGMENT}

The author wishes to thank $T$. Larsen from the Laboratory of Electromagnetic Theory, The Technical Liniversity of Denmark, who set up the initial theory which forms the basis of the present work, for her great help and encouragement with this research. Thanks are due also to J. AppelHansen from the same institution, for many helpful discussions on the subject.

\section{Referexces}

[1] J. Appel-Hansen, "A Van Atta reflector consisting of half-wave dipoles," 1 EEE Trans. Antennas and Propagation, vol. AP-14, pp. 691-700, November 1966.

[2] '2. Larsen, "Reflector arrays," IEEE Trans. Antennas and Propagation, vol. AP-14, pp. 689-693, November 1966.

[3] J. Appel-Hansen, "Lxperimental investigation of a linear Yan Atta reflector," Proc. URSI Symp. on Electromagnelic Wave Theory (Delft, The Netherlands, September 1965). London: Pergamon, 1967, pp. 707-710.

[4] T. Larsen, "Theoretical investigation of Yan Atta reflectors," Proc. LRSI Symp. on Electromagnetic II "ave Theory (Delft, The Netherlands, September 1965). London: Pergamon, 196 $\overline{7}, \mathrm{pp}$. $703-70 \overline{0}$

[5] J. Appel-Hansen, T. Larsen, H. Kurss, and W. K. Kahn, "The influence of the scattered field on the reradiation patter'n of reflector arrays," IEEE T'rans. Antennas and Propagation (Communieations), vol. AP-16, pp. 751-752, November 1968.

[b] H. Kurss and W. K. Kahu, "A note on reflector arrays," IEEE Trans. Antennas and Propagation (Communications), vol. AP15 , pp. 692-693, September 1967.

[i] J. Appel-Hansen, "Coupling in reflector arrays," IEEE Trans. Antennas and Propagation (Communications), vol. AP-16, pp. $759-760$, November 1968.

[8] E. D. Sharp, "Properties of the Van Atta reflector array," Rome Air Development Center, N. Y., Tech. Rept. RADC-TR58-533, ASTIA Doc. AD-148684, April 1958.

[9] S. Davies, "Element vector diagrams for Yagi-Lda antemas," Australian Telecommun. Res., vol. 1, pp. 59-75, November 1967.

[10] D. E. Kerr, Propagation of Short Radio Waies, M. I. T. Rad. Lab. Ser., vol. 13. New York: MeGraw-Hill, 1951, p. 456 .

[11] J. Appel-Hansen, "Optimization of the reradiation pattern of a Ian Atta leflector," Laboratory of Electromagnetic Theory, Technical University of Denmark, Lyngby, USAF Contract AF 61(052)-794, Sci. Rept. 4, June 1966. 